\title{
Review of the uncertainty analysis of groundwater numerical simulation
}

\author{
WU JiChun* \& ZENG XianKui \\ Key Laboratory of Surficial Geochemistry, Ministry of Education, Department of Hydrosciences, School of Earth Sciences and Engineering, State \\ Key Laboratory of Pollution Control and Resource Reuse, Nanjing University, Nanjing 210093, China
}

Received February 18, 2013; accepted May 6, 2013; published online July 31, 2013

\begin{abstract}
Groundwater system is a complex and open system, which is affected by natural conditions and human activities. Natural hydrological processes is conceptualized through relatively simple flow governing equations in groundwater models. Moreover, observation data is always limited in field hydrogeological conditions. Therefore, the predictive results of groundwater simulation often deviate from true values, which is attribute to the uncertainty of groundwater numerical simulation. According to the process of system simulation, the uncertainty sources of groundwater numerical simulation can be divided into model parameters, conceptual model and observation data uncertainties. In addition, the uncertainty stemmed from boundary conditions is sometimes refered as scenario uncertainty. In this paper, the origination and category of groundwater modeling uncertainty are analyzed. The recent progresses on the methods of groundwater modeling uncertainty analysis are reivewed. Furthermore, the researches on the comprehensive analysis of uncertainty sources, and the predictive uncertainty of model outputs are discussed. Finally, several prospects on the deveolpment of groundwater modeling uncetainty analysis are proposed.
\end{abstract}

groundwater modeling, uncertainty analysis, model parameter, conceptual model, observation data

Citation: Wu J C, Zeng X K. Review of the uncertainty analysis of groundwater numerical. Chin Sci Bull, 2013, 58: 3044-3052, doi: 10.1007/s11434-013-5950-8

\section{Conception of the uncertainty of groundwater numerical simulation}

Uncertainty is the inherent characteristics of the objective world, and it is widely presented in human daily life. Generally, uncertainty can be interpreted as the lack of certainty, that is, a future or existing state cannot be described accurately because of limited information [1]. Regional groundwater system is a complex and open system, which is influenced from hydrological and meteorological conditions, geological structure, topography feature, vegetation, and human activities, etc. Furthermore, some properties of groundwater system cannot be measured directly, e.g. hydraulic conductivity and dispersivity, which are estimated indirectly by analyzing input and output measurements [2]. As the natural hydrological processes or groundwater movement is described by a group of simplified water flow governing

\footnotetext{
*Corresponding author (email: jcwu@nju.edu.cn)
}

equations, the predictions of groundwater system always deviate from observations. Therefore, the uncertainty of groundwater simulation is inevitable, which is the main difficulty for describing groundwater flow and solute transport activities accurately [2,3].

Groundwater numerical simulation is the basis for the quantitative analysis of groundwater resources. Successful assessment of groundwater resources depends on reliable and stable groundwater simulation. Therefore, as a result of the uncertainty of groundwater modeling, decision-makers have to face the risk of failure in making decisions [4]. Uncertainty analysis includes studying the origin, transport and producing processes of uncertainties, describing and evaluating the state and characteristics of uncertainties. In addition, the researches on controlling and reducing uncertainties under the current conditions, and making prediction and response for unknown groundwater environment, which are the key problems for uncertainty analysis of groundwater modeling. Therefore, these works about the uncertainty is- 
sues are capable of supplying valuable reference for the management and planning of groundwater resources $[5,6]$.

\section{Sources and classifications of the uncertainty of groundwater numerical simulation}

The result of groundwater modeling is affected by many factors, e.g. the errors arisen from the construction of groundwater conceptual model, the deviations caused by the approximate solution of groundwater mathematic model. Furthermore, the scarcity and observation error of measurement data enhance the generation of modeling uncertainty. In addition, trapped in unknown interaction mechanism between various factors, it is hard to separate each part of uncertainty sources explicitly, and describe it independently.

The uncertainty sources of groundwater or hydrological model are classified inconsistently by different authors. Yen et al. [7] partitioned the modeling uncertainty into 5 parts: (1) the natural uncertainty caused by the inherent randomness of natural process; (2) the model uncertainty stemmed from defective model which is not able to represent the real physical processes; (3) the uncertainty of model parameter; (4) the uncertainty derived from observation error; and (5) the operating uncertainty caused by human factors. Van Asselt [8] proposed a classification upon the decision support management of groundwater model, the uncertainty sources could be cognized at three levels that are generation location, managing level and natural quality respectively. Liu and Shu [9] pointed out that, according to discipline nature, the source could be interpreted as stochastic, fuzzy, gray, and unknown uncertainties. In addition, according to the reason for generating, the source could be partitioned as the uncertainties that caused by natural processes, human activities and cognizing ability. Merz and Thieken [10] treated the modeling uncertainty as two types, aleatory and epistemic uncertainties. Furthermore, these researches [11-14], etc., summarized and discussed the uncertainty of groundwater numerical simulation from different points of view.

In recent years, researches on the uncertainty analysis of groundwater simulation emerge in large numbers. In order to obtain simplified and reasonable statistics for modeling uncertainty, and satisfy the actual needs of hydrogeological workers, more and more researchers, e.g. [3,6,15-21], accepted to use a framework to analyze groundwater modeling uncertainty. In general, according to the logical process of groundwater modeling, the uncertainty stems from three sources which are model parameter, conceptual model (or model structure) and observation data, respectively.

Generally, the establishment of a groundwater numerical model requires inputting a set of parameters, e.g. the hydraulic conductivity, transmissivity, specific yield, storage coefficient and dispersivity. Hydrogeological data is always limited in the field, therefore, the parameter uncertainty is derived from unreasonable parameter division, the temporal and spatial variability and the scaling effect of parameters. Next, conceptual model is the basis of numerical model, and actual hydrogeological conditions are often simplified incorrectly by groundwater conceptual model $[6,22]$. Therefore, the model structure uncertainty is influenced by many factors, including the incorrect setting of model aquifers (location, type, number of layer, distribution, etc.), unreasonable estimation of groundwater model's boundary conditions and sources and sinks, and the approximation of special groundwater processes. Furthermore, for groundwater simulation, observation data is often used for two aspects, the input data used for building groundwater model, and the conditioning data or calibration data applied to calibrate model. The observation uncertainty stems from a very wide range, including the error caused by the stochastic distribution of the observed variable, the sampling error of the observed variable, indirect measurement error, the error of measuring device, and human recording error, etc. In addition, some authors regarded the uncertainty of model boundary conditions as the fourth uncertainty which is referred as scenario uncertainty [23,24].

\section{Uncertainty analysis methods for groundwater numerical simulation}

According to the inherent structure's statistical properties and the input of a system, the most direct method for assessing the output uncertainty is to derive the statistical information of output directly [25]. However, this approach is restrained in two aspects, one is the troubles in mathematics and numerical solution when deriving statistical result, the other is actual condition that the detailed statistical properties on the system structure and input are unknown usually. Therefore, the direct method is always infeasible in practical application.

Probability theory is traditionally used for the uncertainty analysis of hydrological processes and other fields of science. Recently, many researchers $[1,25]$ believe that the uncertainty can be effectively treated in a broad perspective, and the statistical information will be an important approach. According to the analysis methods for the uncertainties from model parameter, model structure and observation data, this paper summarized and discussed the developments of uncertainty analysis.

\subsection{Uncertainty analysis of groundwater model parameters}

As the groundwater model is used for groundwater resources simulation and prediction, how to choose appropriate model parameters becomes the most immediate problem which model users have to face. Moreover, the simulated 
result depends on model parameters when the model structure is fixed. Hence, parametric uncertainty received the attention of hydrogeologists firstly, and it is an important part of groundwater modeling uncertainty. At present, the most popular and feasible methods for parametric uncertainty including generalized likelihood uncertainty estimation (GLUE), Markov Chain Monte Carlo (MCMC), Bayesian recursive estimation (BaRE), etc.

Beven and Binley [26] firstly proposed the concept of equifinality in the uncertainty analysis of hydrological model. And then, they developed a generalized likelihood uncertainty estimation (GLUE) method based on regionalized sensitivity analysis. The key idea of GLUE method is getting rid of the framework of global optimal parameter solution. Moreover, GLUE believes that the reason for leading the performance of a groundwater model is not a single parameter, but the combination of model parameters [26,27]. Firstly, model parameters are defined in prescribed ranges, parameter set is randomly sampled from prior probability distributions, and the numerical model is run by Monte Carlo method. Secondly, an appropriate likelihood function is selected to evaluate the goodness of fit between simulated results and observations. The parameter set with the closer simulation to observation is given a higher confidence, and it is assigned with a likelihood value or likelihood weight. In addition, if the likelihood value of a simulation result is below a prescribed critical value, the corresponding parameter set is regarded as a non-behavioral or unacceptable parameter, and its likelihood value is set to 0 . Lastly, all the likelihood values are normalized, the posterior probability distributions of model parameters and outputs are obtained by analyzing the retained samples.

GLUE has the advantage of simple structure, easy operation, and wide applicability, so that it can be applied to the uncertainty analysis of various water resources and environmental models [15]. GLUE has been widely used for many purposes, such as precipitation-runoff model [27], distributed basin hydrological model [28], soil erosion model [29], groundwater model [3], unsaturated zone model [30], flood model [31]. Nevertheless, while GLUE is widely recognized in hydrology simulation, the critical and controversial voices are also cannot be ignored. GLUE is constructed based on Monte Carlo technique, the efficiency and reliability of sampling algorithm is the key of method. Traditionally, GLUE takes advantage of a uniform sampling algorithm to obtain parameters' posterior distributions. However, because of the ineffective sampling technique, this method requires a huge number of simulations to obtain the convergence of Monte Carlo simulation [15]. Furthermore, for complex and high-dimensional uncertainty issues, it is likely to generate unreliable and inconsistent result. From the points of extracting information and evaluating uncertainty for hydrological forecast, Mantovan and Todini $[32,33]$ thought that GLUE is not a formal Bayesian inference method, it is lack of consistency and coherence. In the same way, Hassan et al. [3] and Beven et al. [34,35] made corresponding responses for these critism from the views of practical application.

Markov Chain Monte Carlo (MCMC) is a dynamic sampling technique. By constructing a Markov Chain with stable density distribution, the probability distribution space of target function is sufficiently searched in the process of evolving Markov Chain [2,15]. The searching process is constructed by two functions which are proposal and acceptance functions. Proposal function (or proposal distribution) is used for generating alternative samples of parameter set, and whether the parameter sample is accepted or rejected depends on acceptance function (or transition function). Sampling algorithm is the core of MCMC, which determines the sampling efficiency and reliability of uncertainty analysis. In addition, various MCMC methods can be established by designing different sampling techniques according to specific objective [36].

Recently, the common used sampling techniques can be divided into two categories, single-chain and multi-chains techniques. Single-chain technique is developed in early MCMC methods. Metropolis [37] firstly raised the concept of MCMC sampling, and proposed the Metropolis algorithm to simulate energy level of atoms in crystal structure. Based on Metropolis algorithm, Hastings [38] developed Metropolis-Hastings(M-H) algorithm which is able to make use of any form of transition function, and meet the requirement of detailed balance. Aim at the selection of proposal function, Haario et al. [39] developed an adaptive proposal distribution (AP) algorithm, AP is operated by a normal distribution of which the mean and variance are calculated by retained samples. Based on AP algorithm, Haario et al. [40] developed an Adaptive Metropolis (AM) algorithm, with respect to $\mathrm{AP}, \mathrm{AM}$ is superior in updating the mean and covariance of proposal distribution by using previous sampling information based on a regression formula. Moreover, other single-chain techniques include MHBC [41], MHSS and MHBU [42], Gibbs algorithm [43], etc.

Based on the optimization algorithm SCE-UA [44], Vrugt et al. [36] developed a multi-chain evolving algorithm, Shuffled Complex Evolution Metropolis algorithm (SCEM) which assembles the advantages of M-H algorithm, controlling random search, competition evolution and shuffled complex evolution. The convergence rate is improved by adopting multiple parallel Markov chains, and exchanging searching information between chains. Braak [45] reported a genetic algorithm based differential evolution Markov Chain (DE-MC). This is a multi-chain parallel evolution technique by combining MCMC and differential evolution method. Based on DE-MC, Vrugt et al. [46] proposed a differential evolution adaptive Metropolis algorithm (DREAM). This method generates a proposal sample based on the difference of one (or several) couple of parameter samples. In addition, the adopted strategies of DREAM include sampling from a group of updated random subspaces, 
estimating the probability distribution of the cross probabilities of random subspaces. Therefore, the convergence rate of DREAM is improved significantly. Moreover, the other multi-chain sampling technique include $\operatorname{DREAM}_{(\mathrm{D})}$ [47] and DREAM $(\mathrm{ZS})$ [48], NSMC [49], etc.

With the development of mathematic statistics methods, the sampling algorithm of MCMC is improved continuously. In general, MCMC method is superior in strong flexibility, and high reliability in various environmental models' uncertainty analysis. Especially, MCMC method has a good performance on complex uncertainty issues which include high nonlinear, high dimensional and multimodal probability distribution $[2,15]$. Furthermore, MCMC method is inferior in huge computing time-consuming requirement. In addition, MCMC method is restricted in the application of parallel computing techniques because of its logic computing characteristics.

For comparing and analyzing parameter uncertainty methods, GLUE and MCMC (DREAM) are compared based on a synthetic groundwater flow model [6] in this study. This model is a 3-dimensional transient groundwater field constructed by three heterogeneous layers, which are regarded as unconfined aquifer, aquitard, and confined aquifer from top to bottom. Two cases of parameter uncertainty that include 4-dimensional and 8-dimensional parameter spaces (groundwater models constructed with 4 and 8 unknown parameters) are considered, and these two methods are compared for parameter identification and output prediction.

Figures 1 and 2 show the predictions of groundwater budget terms of the 4-dimensional groundwater model based on GLUE and DREAM respectively. The results indicate that the predictive variables can be appropriately predicted by both GLUE and DREAM methods. Figures 3 and 4 represent the predictions of budget terms of the 8-dimensional groundwater model based on GLUE and DREAM respectively. The results show that the predictive variables' posterior distributions are almost uniformly distributed in the ranges of corresponding prior distributions for GLUE method. Therefore, the posterior distributions cannot provide any predictive information, which represents the prediction failure of GLUE. By contrast, the budget terms are appropriately predicted by DREAM method (except for the OutRiv). In addition, the parameter identifications of GLUE and DREAM have the similar characteristics with output predictions, which are not shown here for lack of space. Therefore, GLUE method is only usable for low-dimensional parametric uncertainty analysis, and DREAM is more accurate and stable for high-dimensional and complex uncertainty problems.

Thiemann et al. [50] firstly proposed the theory framework of Bayesian recursive estimation (BaRE) which can
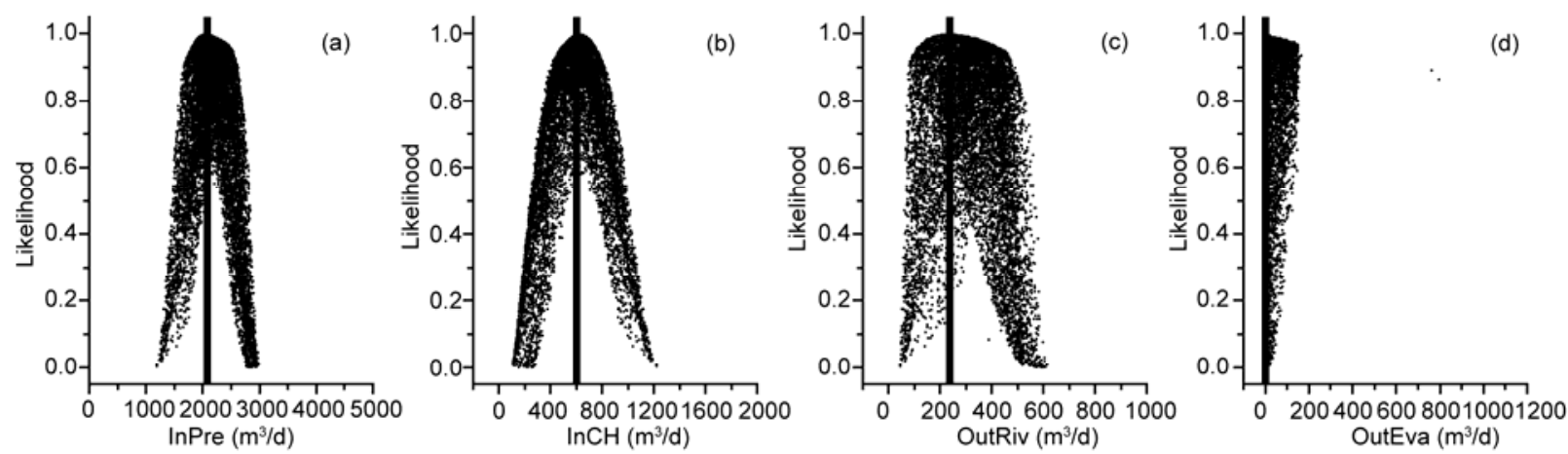

Figure 1 Likelihood response surfaces of groundwater budget terms for the 4-dimensional model predicted by GLUE, the vertical line denotes corresponding true value, and the horizontal axis labels groundwater budget terms.
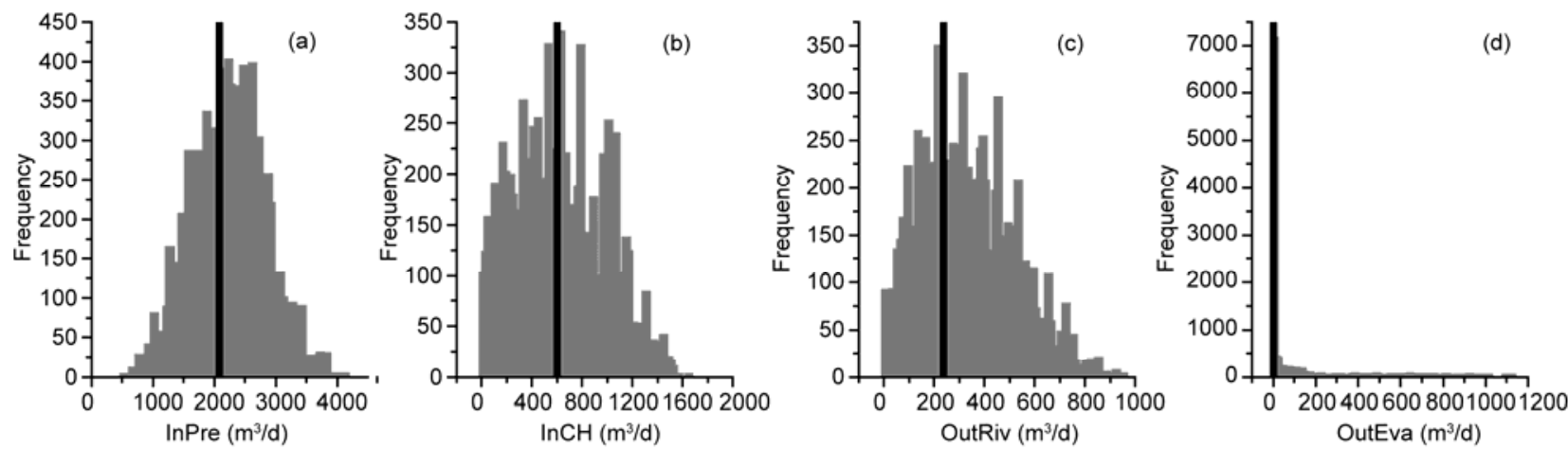

Figure 2 Frequency distributions of groundwater budget terms for the 4-dimensional model predicted by DREAM, the vertical line denotes corresponding true value, and the horizontal axis labels groundwater budget terms. 

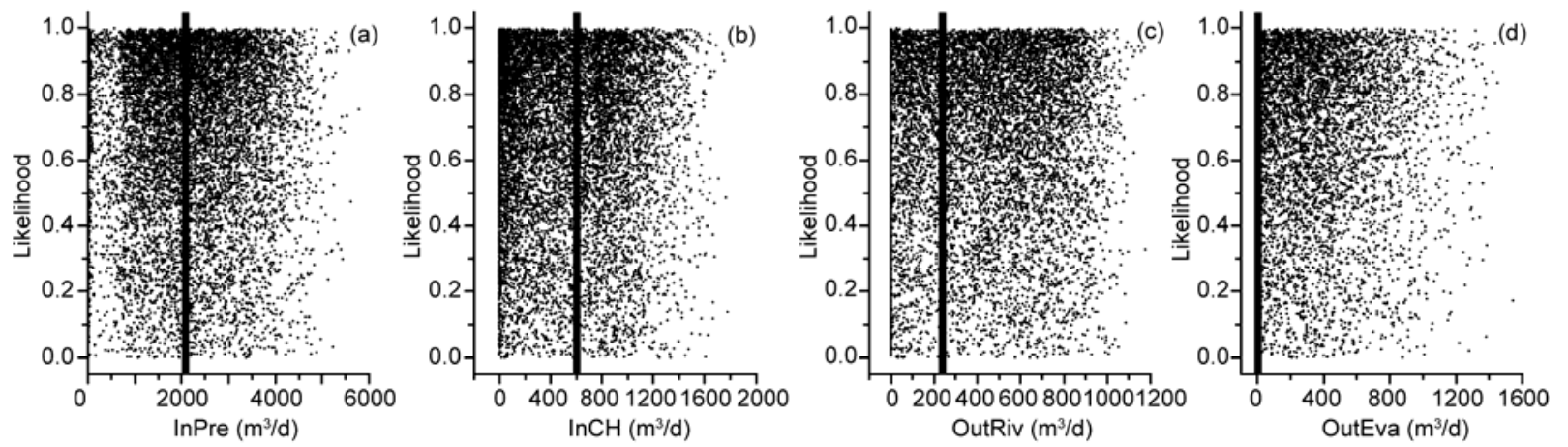

Figure 3 Likelihood response surfaces of groundwater budget terms for the 8-dimensional model predicted by GLUE, the vertical line denotes corresponding true value, and the horizontal axis labels groundwater budget terms.
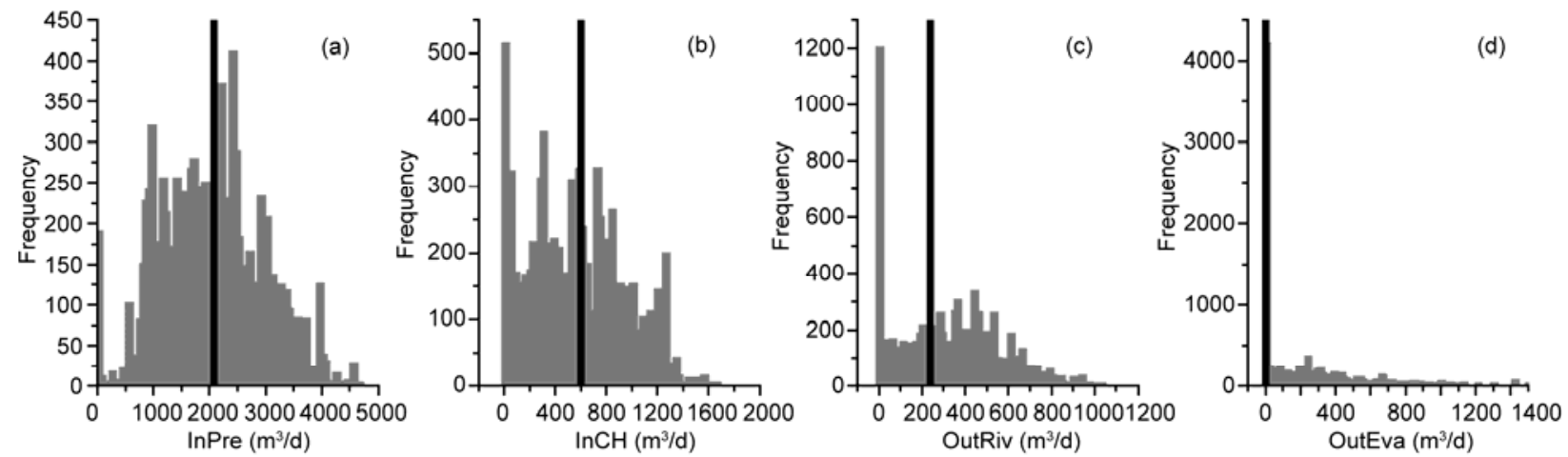

Figure 4 Frequency distributions of groundwater budget terms for the 8-dimensional model predicted by DREAM, the vertical line denotes the corresponding value, and the horizontal axis labels groundwater budget terms.

treat the uncertainties of hydrological model parameters and outputs simultaneously. The model output is predicted in the form of probability, and the new added observations are incorporated into prediction by recursive updating. Moreover, the common used analysis software or code also include UCODE [51] and PEST [52], etc.

\subsection{Uncertainty analysis of groundwater conceptual model}

The uncertainty of groundwater conceptual model (model structure) had not given enough attention in traditional uncertainty analysis of groundwater modeling. Usually, groundwater system is represented by only one hydrogeological conceptual model [6]. It is prone to obtain incorrect simulation results based on a single model structure by neglecting the possible of others conceptual models. In addition, the model's predictive power is overestimated, and output is likely predicted with statistical deviation. Especially, it is riskier to ignore conceptual model uncertainty when predictive variable is not applied to model calibration. In addition, the results of [53] demonstrated that the correctness of groundwater conceptual model cannot be assured by an excellent model calibration. Based on a synthetical groundwater model, the result of [6] showed that the $5 \%-30 \%$ of predictive uncertainty is derived from model structure. Therefore, conceptual model uncertainty should be given sufficient attention for groundwater uncertainty analysis.

As the development of uncertainty analysis in groundwater conceptual model, the multi-model method has become an important theory in treating groundwater modeling uncertainty. The groundwater system is represented by a set of conceptual models which are weighted by their corresponding performance on reproducing the groundwater system $[6,54]$. In addition, the behavior of unknown groundwater system is described by the combination of outputs of alternative models. In general, multi-model analysis includes following steps: (1) constructing a group of plausible conceptual models based on prior information; (2) calibrating these alternative conceptual models by obtained conditioning data; (3) weighting or ranking these conceptual models by using a criterion; (4) removing these models with significantly unreasonable performances; and (5) ensemble prediction by combining the weighted predictions of retained conceptual models.

Poeter and Anderson [55] proposed a Kullback-Leibler information based multi-model theory, this method can evaluate the weight of alternative models, but the prior information cannot be formally incorporated into assembled predictions. Refsgaard et al. [56] developed a pedigree analysis method to assess conceptual model uncertainty, this 
method is able to integrate various kinds of prior knowledge. However, each part of predictive uncertainty cannot be delineated quantitatively. Bayesian Model Averaging (BMA) method is firstly proposed by $[57,58]$, this method infers the posterior probabilities of alternative conceptual models based on Bayesian inference, and each part of predictive uncertainty can be described separately. The formula of BMA is given as:

$$
p(\Delta \mid \boldsymbol{Z})=\sum_{k=1}^{K} p\left(\Delta \mid \boldsymbol{Z}, M_{k}\right) p\left(M_{k} \mid \boldsymbol{Z}\right),
$$

where $\Delta$ denotes a predictive variable, $p(\Delta \mid Z)$ is the ensemble probability of $\Delta$, and $p\left(\Delta \mid Z, M_{k}\right)$ represents the probability of $\Delta$ given observation $\boldsymbol{Z}$ and model $M_{k} \cdot p\left(M_{k} \mid Z\right)$ is the posterior probability of model $M_{k}$, which can be computed using Bayesian theorem:

$$
p\left(M_{k} \mid \boldsymbol{Z}\right)=\frac{p\left(\boldsymbol{Z} \mid M_{k}\right) p\left(M_{k}\right)}{\sum_{i=1}^{K} p\left(\boldsymbol{Z} \mid M_{i}\right) p\left(M_{i}\right)},
$$

where $p\left(M_{k}\right)$ denotes the prior probability of model $M_{k}$, and $p\left(Z \mid M_{k}\right)$ is the integrated likelihood measure of conceptual model $M_{k}$.

Conceptual model's posterior probability is obtained by combining conceptual model's prior probability and integrated likelihood value $p\left(Z \mid M_{k}\right)$ which indicates the performance on reproducing groundwater observations [23,58]. Moreover, the variance of groundwater model's prediction is divided into within-model and between-model variances which represent the uncertainties of model parameter and structure respectively $[6,24]$. According to the method for calculating conceptual model's integrated likelihood value, BMA methods can be divided into two broad categories: the Monte Carlo based BMA method (MC-BMA) and the information criteria (or model selection criteria) based BMA method (IC-BMA) [14].

Kullback-Leibler (K-L) information is the base of model selection theory. When information criteria is used for selecting models, each conceptual model will obtain a K-L value which represents the loss of information as the real groundwater system is represented by this model [56]. Nevertheless, K-L value cannot be calculated directly because of the unknown groundwater system [56]. Therefore, a series of model selection criteria are developed for estimating $\mathrm{K}-\mathrm{L}$ value, e.g. Akaike Information Criterion (AIC) [59], AICc [60], BIC [61], HQC [62], KIC [63].

IC-BMA is the main idea for current multi-model averaging methods. Neuman and Wierenga [64] proposed a suit of strategies for constructing and selecting conceptual models, assembling models' outputs and making optimum predictions. However, this method concerns the optimum prediction, which is lack of formality. And then, Neuman [65] proposed a KIC based maximum likelihood Bayesian modeling average (MLBMA) to overcome this defect. MLBMA can integrate the information on field conditions and observations, and the final outcome depends on the combination of model outputs and prior information. Ye et al. [66] applied MLBMA to analyze the groundwater flow modeling uncertainty of the unsaturated fracture tuff at a testing site, Apache Leap, middle of Arizona. Tsai and Li [67] used BIC-BMA to evaluate the uncertainty of seawater intrusion-groundwater management model at Baton Rouge, Louisiana. Furthermore, Refs. [24,68,69], etc. also have studied conceptual model uncertainty based on IC-BMA method. In addition, the software Multi-Model Analysis (MMA) [70] is a convenient and efficient tool for conceptual model's uncertainty analysis.

MC-BMA method calculates conceptual model's integrated likelihood value $p\left(Z \mid M_{k}\right)$ by Monte Carlo simulation which is applied to inverse model's parameter space. Rojas et al. [6] firstly proposed GLUE based BMA method, for which GLUE is used for estimating conceptual model's parametric uncertainty. The summation of all retained samples' weights is regarded as conceptual model's integrated likelihood value. In addition, Rojas et al. [23] pointed that, for IC-BMA method, the application of information criteria includes a step of model calibration. Thus, model structure's deviation could be compensated by model calibration, which will cause statistical bias of conceptual model's posterior probability. By contrast, MC-BMA determines posterior weight based on the likelihood distribution of corresponding conceptual model and its prior probability. Therefore, MC-BMA prevents multi-model average prediction from the erosion of biased parameter estimation.

MC-BMA method is not popular in current multi-model researches, and it is mainly seen in Rojas's studies, such as [6,23,71-73]. Furthermore, Raftery et al. [74] proposed an expectation maximization (EM) method to solve the weight and variance of conceptual models iteratively. However, EM-BMA assumes that model's prediction follows normal distribution, and it is hard to assure that EM algorithm convergences to global optimum model's weight and variance. Harp and Vesselinov [75] proposed an approach of hydrogeological acceptance probability to analysis the possibility of a groundwater model, and the hydrogeological acceptance probability is estimated to the sample mean of a Bernoulli distribution.

\subsection{Uncertainty analysis of groundwater observation data}

Observation error includes system and random errors which are produced in the processes of measuring, collecting, recording, storing, and importing data. When groundwater model is calibrated and verified based on a group of biased observation data, the uncertainties related to model output, parameter inversion, output prediction, etc., are regarded as observation error. In addition, observation error is always merged with other sources, such as model parameter and structure uncertainties, to affect model output simultaneously. Therefore, it is difficult to separate these uncertain- 
ties from integrated affection accurately.

Generally, groundwater model observation error has not given enough attention with respect to model parameter and conceptual model uncertainties. Observation data is always assume to be accurate, or given a simplified error structure $[76,77]$. Moreover, groundwater model observation uncertainty is assessed combined with the uncertainty analysis of model parameter and (or) structure. Furthermore, groundwater is a special system, the simulation errors stem from multiple sources, and data scarcity is the especial factor which enhances the uncertainty of groundwater numerical simulation. Therefore, the residuals will often have a complicated structure that is hard to delineate or interpret.

At present, observation uncertainty is usually assessed by comprehensive evaluation methods. The common used methods include Bayesian forecasting system (BFS) [78], integrated Bayesian uncertainty estimator (IBUNE) [18], Bayesian total error analysis (BATEA) [79,80], and data fusion method [73], etc. Troldborg et al. [73] proposed an assembled method which combines Bayesian model averaging, Bayesian geostatistics method and Kalman ensemble generator to account for conceptual model and measurement uncertainties. The method was used for assessing the uncertainty of mass discharge from a contaminated site located at northern Copenhagen. In addition, the observation error is assumed to be normal and independent with zero mean and a fixed covariance matrix. Renard et al. [21] evaluated the predictive uncertainty of a conceptual rainfall-runoff model based on BATEA. Furthermore, the total uncertainty was divided into input and structure uncertainties, and they were described and assessed quantitatively. Renard et al. [77] made a comparison between IBUNE and BATEA, and drew a conclusion that these two methods are both constructed based on the hierarchical generalized processing of input uncertainty. Moreover, for the first type of IBUNE, the likelihood function and posterior distribution are predictive variable's stochastic functions, which is inconsistent with the standard essential condition of probability density function. In addition, the second type of IBUNE is inferior in sampling efficiency and convergence rate.

\section{Conclusion and prospect}

In conclusion, the uncertainty analysis of groundwater numerical simulation has received extensive researches and attention, and produced a series of valuable achievements. Nevertheless, most of researches rest on theory analysis or simplified artificial case, which are not enough powerful and reliable for practical application, such as the risk assessment of a contaminant site's remediation, water resources management of a watershed under global climate change, etc. Therefore, the following aspects should give sufficient attention:

(1) Effective and reliable sampling algorithm. Monte
Carlo method is always treated as the framework of uncertainty analysis of groundwater modeling, and groundwater numerical model has to be run for tens of thousands times to achieve output convergence. Thus, the heavy computing time-consuming is the main restriction for this kind of methods. Especially, for some field site groundwater system, the time-consuming of single numerical model is also not ignorable because of the complex hydrogeological conditions. Therefore, an effective and reliable sampling algorithm can directly overcome this problem, which makes it possible to apply the uncertainty analysis theory to some practice cases.

(2) Determination of conceptual model's prior probability. Conceptual model's prior probability is determined based on previous research data or expertise, which is belongs to the scope of subjective judgment. Moreover, conceptual model's posterior probability and averaging prediction are significantly influenced by prior probability. Therefore, the quantity and quality of current prior information is crucial for groundwater modeling uncertainty analysis. In addition, geophysical survey methods (e.g. high-density resistivity method, induced polarization method, seismic method) are able to provide plentiful and full-scale detection information for regional hydrogeological conditions at a low cost.

(3) Calculating method for conceptual model's posterior probability. The multi-model averaging prediction depends on conceptual model's posterior probability. However, the calculating methods for model's posterior probability have not received a consistent acknowledgement, and different calculating methods will cause diverse assessment results. Therefore, it is crucial to adopt a reasonable method to calculate conceptual model's integrated likelihood value.

(4) Integrated assessment of the uncertainty sources of groundwater modeling. The uncertainty sources are always assessed separately, and some sources are often simplified or ignored in present groundwater modeling uncertainty analysis. Moreover, some methods used in the uncertainty assessment of hydrologic models are not applicable for groundwater modelling. These hydrologic models are always referring as precipitation-runoff models, the error in precipitation is considered as the main uncertainty source of model input, but that is not truth for groundwater modelling. Groundwater numerical simulation is influenced by more factors. Therefore, an integrated evaluation method is required to account for all uncertainty sources simultaneously for obtaining accurate and reliable assessment result.

(5) Quantitative assessment of the uncertainty of groundwater numerical simulation. Variance is currently used as the measurement method for groundwater modeling uncertainty. However, variance method is only appropriate for univariate probability distribution, it is extended as a disperse matrix for multivariate distribution. Thus, the target probability distribution cannot be uniquely represented by a scalar function. Moreover, variance is not a general 
method for representing the concentrate extent of a probability distribution, except for normal distribution. By contrast, information entropy method satisfies with all the derivative properties and Shannon rules of an uncertainty measurement function [81]. In addition, information entropy method is constructed based on stable theory foundation, and it is applicable to multivariate and any forms of probability distributions. Therefore, it is feasible to apply information entropy theory to assess the uncertainty of groundwater numerical simulation.

This work was supported by the National Natural Science Fundation of China (41172207, 41071018, 41030746, 40725010 and 40730635).

1 Zadeh L A. Toward a generalized theory of uncertainty (GTU)—An outline. Inform Sci, 2005, 172: 1-40

2 Hassan A E, Bekhit H M, Chapman J B. Using Markov Chain Monte Carlo to quantify parameter uncertainty and its effect on predictions of a groundwater flow model. Environ Modell Softw, 2009, 24: 749-763

3 Hassan A E, Bekhit H M, Chapman J B. Uncertainty assessment of a stochastic groundwater flow model using GLUE analysis. J Hydrol, 2008, 362: 89-109

4 Zeng X K, Wang D, Wu J C. Sensitivity analysis of the probability distribution of groundwater level series based on information entropy. Stoch Env Res Risk A, 2012, 26: 345-356

5 Ajami N K, Hornberger G M, Sunding D L. Sustainable water resource management under hydrological uncertainty. Water Resour Res, 2008, 44: W11406

6 Rojas R, Feyen L, Dassargues A. Conceptual model uncertainty in groundwater modeling: Combining generalized likelihood uncertainty estimation and Bayesian model averaging. Water Resour Res, 2008, 44: W12418

7 Yen B C, Cheng S T, Melching C S. Stochastic and Risk Analysis in Hydraulic Engineering. Littleton: Water Resources Publications, 1986

8 Van Asselt M. Perspectives on Uncertainty and Risk: The PRIMA Approach to Decision Support. Dordrecht: Kluwer Academic Publishers, 2000

9 Liu P G, Shu L C. Uncertainty on numerical simulation of groundwater flow in the riverside well field (in Chinese). J Jilin Univ (Earth Science Edition), 2008, 38: 639-644

10 Merz B, Thieken A H. Flood risk curves and uncertainty bounds. Nat Hazards, 2009, 51: 437-458

11 Yang P H, Yuan D X, Yuan W H, et al. Formations of groundwater hydrogeochemistry in a karst system during storm events as revealed by PCA. Chin Sci Bull, 2010, 55: 1412-1422

12 Helton J C, Oberkampf W L. Alternative representations of epistemic uncertainty. Reliab Eng Syst Safe, 2004, 85: 1-10

13 Katz R W, Parlange M B, Naveau P. Statistics of extremes in hydrology. Adv Water Resour, 2002, 25: 1287-1304

14 Singh A, Mishra S, Ruskauff G. Model averaging techniques for quantifying conceptual model uncertainty. Ground Water, 2010, 48: 701-715

15 Blasone R S, Vrugt J A, Madsen H, et al. Generalized likelihood uncertainty estimation (GLUE) using adaptive Markov Chain Monte Carlo sampling. Adv Water Resour, 2008, 31: 630-648

16 Wu J C, Lu L, Tang T. Bayesian analysis for uncertainty and risk in a groundwater numerical model's predictions. Hum Ecol Risk Assess, 2011, 7: 1310-1331

17 Jin X L, Xu C Y, Zhang Q, et al. Parameter and modeling uncertainty simulated by GLUE and a formal Bayesian method for a conceptual hydrological model. J Hydrol, 2010, 383: 147-155

18 Ajami N K, Duan Q Y, Sorooshian S. An integrated hydrologic Bayesian multimodel combination framework: Confronting input, parameter, and model structural uncertainty in hydrologic prediction. Water Resour Res, 2007, 43: W01403

19 Zeng X K, Wang D, Wu J C, et al. Reliability analysis of the groundwater conceptual model. Hum Ecol Risk Assess, 2013, 19: 515-525

20 Xu X W, Li B W, Wang X J. Progress in study on irrigation practice with saline groundwater on sandlands of Taklimakan Desert Hinterland. Chin Sci Bull, 2006, 51: 161-166

21 Renard B, Kavetski D, Kuczera G, et al. Understanding predictive uncertainty in hydrologic modeling: The challenge of identifying input and structural errors. Water Resour Res, 2010, 46: W05521

22 Refsgaard J C, Christensen S, Sonnenborg T O, et al. Review of strategies for handling geological uncertainty in groundwater flow and transport modeling. Adv Water Resour, 2012, 36: 36-50

23 Rojas R, Kahunde S, Peeters L, et al. Application of a multimodel approach to account for conceptual model and scenario uncertainties in groundwater modelling. J Hydrol, 2010, 394: 416-435

24 Ye M, Pohlmann K F, Chapman J B, et al. A model-averaging method for assessing groundwater conceptual model uncertainty. Ground Water, 2010, 48: 716-728

25 Montanari A. What do we mean by 'uncertainty'? The need for a consistent wording about uncertainty assessment in hydrology. Hydrol Process, 2007, 21: 841-845

26 Beven K, Binley A. The future of distributed models-Model calibration and uncertainty prediction. Hydrol Process, 1992, 6: 279-298

27 Beven K, Freer J. Equifinality, data assimilation, and uncertainty estimation in mechanistic modelling of complex environmental systems using the GLUE methodology. J Hydrol, 2001, 249: 11-29

28 Vazquez R F, Beven K, Feyen J. GLUE based assessment on the overall predictions of a MIKE SHE application. Water Resour Manag, 2009, 23: 1325-1349

29 Brazier R E, Beven K J, Anthony S G, et al. Implications of model uncertainty for the mapping of hillslope-scale soil erosion predictions. Earth Surf Proc Land, 2001, 26: 1333-1352

30 Mertens J, Madsen H, Feyen L, et al. Including prior information in the estimation of effective soil parameters in unsaturated zone modelling. J Hydrol, 2004, 294: 251-269

31 Aronica G, Hankin B, Beven K. Uncertainty and equifinality in calibrating distributed roughness coefficients in a flood propagation model with limited data. Adv Water Resour, 1998, 22: 349-365

32 Mantovan P, Todini E. Hydrological forecasting uncertainty assessment: Incoherence of the GLUE methodology. J Hydrol, 2006, 330: 368-381

33 Mantovan P, Todini E, Martina M L V. Reply to comment by Keith Beven, Paul Smith and Jim Freer on "Hydrological forecasting uncertainty assessment: Incoherence of the GLUE methodology". J Hydrol, 2007, 338: 319-324

34 Beven K, Smith P, Freer J. Comment on "Hydrological forecasting uncertainty assessment: Incoherence of the GLUE methodology" by Pietro Mantovan and Ezio Todini. J Hydrol, 2007, 338: 315318

35 Beven K, Smith P, Freer J. So just why would a modeller choose to be incoherent? J Hydrol, 2008, 354: 15-32

36 Vrugt J A, Gupta H V, Bouten W, et al. A Shuffled Complex Evolution Metropolis algorithm for optimization and uncertainty assessment of hydrologic model parameters. Water Resour Res, 2003, 39: Artn 1201

37 Metropolis N, Rosenbluth A W, Rosenbluth M N, et al. Equation of state calculations by fast computing machines. J Chem Phys, 1953, 21: 1087-1092

38 Hastings W K. Monte Carlo sampling methods using Markov chains and their applications. Biometrika, 1970, 57: 97-109

39 Haario H, Saksman E, Tamminen J. Adaptive proposal distribution for random walk Metropolis algorithm. Comput Stat, 1999, 14: 375-395

40 Haario H, Saksman E, Tamminen J. An adaptive Metropolis algorithm. Bernoulli, 2001, 7: 223-242

41 Bates B C, Campbell E P. A Markov chain Monte Carlo scheme for parameter estimation and inference in conceptual rainfall-runoff 
modeling. Water Resour Res, 2001, 37: 937-947

42 Marshall L, Nott D, Sharma A. A comparative study of Markov chain Monte Carlo methods for conceptual rainfall-runoff modeling. Water Resour Res, 2004, 40: W02501

43 Gelfand A E, Hills S E, Racine-Poon A, et al. Illustration of Bayesian inference in normal data models using Gibbs sampling. J Am Stat Assoc, 1990, 85: 972-985

44 Duan Q Y, Sorooshian S, Gupta V. Effective and efficient global optimization for conceptual rainfall-runoff models. Water Resour Res, 1992, 28: 1015-1031

45 Braak C. A Markov Chain Monte Carlo version of the genetic algorithm Differential Evolution: Easy Bayesian computing for real parameter spaces. Stat Comput, 2006, 16: 239-249

46 Vrugt JA, ter Braak C J F, Diks C G H, et al. Accelerating Markov Chain Monte Carlo simulation by differential evolution with self-adaptive randomized subspace sampling. Int J Nonlin Sci Num, 2009, 10: 273-290

47 Vrugt J A. DREAM(D): An adaptive markov chain monte carlo simulation algorithm to solve discrete, noncontinuous, posterior parameter estimation problems. Hydrol Earth Syst Sci, 2011, 8: 4025-4052

48 Laloy E, Vrugt J A. High-dimensional posterior exploration of hydrologic models using multiple-try $\operatorname{DREAM}((\mathrm{ZS}))$ and highperformance computing. Water Resour Res, 2012, 48: W01526

49 Yoon H, Hart D B, McKenna S A. Parameter estimation and predictive uncertainty in stochastic inverse modeling of groundwater flow: Comparing null-space monte carlo and multiple starting point methods. Water Resour Res, 2013, 49: 536-553

50 Thiemann M, Trosset M, Gupta H, et al. Bayesian recursive parameter estimation for hydrologic models. Water Resour Res, 2001, 37: 2521-2535

51 Poeter E P, Hill M C. UCODE, a computer code for universal inverse modeling. Comput Geosci, 1999, 25: 457-462

52 Doherty J E. PEST, Model-independent Parameter Estimation, User Manual 5th Edition, 2010

53 Bredehoeft J. The conceptualization model problem-surprise. Hydrogeol J, 2005, 13: 37-46

54 Troldborg L, Refsgaard J C, Jensen K H, et al. The importance of alternative conceptual models for simulation of concentrations in a multi-aquifer system. Hydrogeol J, 2007, 15: 843-860

55 Poeter E, Anderson D. Multimodel ranking and inference in ground water modeling. Ground Water, 2005, 43: 597-605

56 Refsgaard J C, van der Sluijs J P, Brown J, et al. A framework for dealing with uncertainty due to model structure error. Adv Water Resour, 2006, 29: 1586-1597

57 Draper D. Assessment and propagation of model uncertainty. J Roy Stat Soc B Met, 1995, 57: 45-97

58 Kass R E, Raftery A E. Bayes factors. J Am Stat Assoc, 1995, 90: 773-795

59 Akaike H. A new look at the statistical model identification. IEEE Trans Automat Contr, 1974, 19: 716-723

60 Hurvich C M, Tsai C L. Regression and time series model selection in small samples. Biometrika, 1989, 76: 297-307

61 Schwarz G. Estimating the dimension of a model. Ann Statist, 1978, 6: 461-464

62 Hannan E J, Quinn B G. The Determination of the order of an autoregression. J Roy Stat Soc Ser B-Stat Met, 1979, 41: 190-195

63 Kashyap R L. Optimal choice of AR and MA parts in autoregressive moving average models. IEEE Trans Patt Anal Mach Int, 1982, 4: 99-104

64 Neuman S P, Wierenga P J. A comprehensive strategy of hydrogeologic modeling and uncertainty Analysis for nuclear facilities and sites. NUREG/CR-6805, u.s. Nucl Regul Comm, Washington D C, 2003

65 Neuman S P. Maximum likelihood Bayesian averaging of uncertain model predictions. Stoch Env Res Risk A, 2003, 17: 291-305

66 Ye M, Neuman S P, Meyer P D. Maximum likelihood Bayesian averaging of spatial variability models in unsaturated fractured tuff. Water Resour Res, 2004, 40: W05113

67 Tsai FTC, Li X. Inverse groundwater modeling for hydraulic conductivity estimation using Bayesian model averaging and variance window. Water Resour Res, 2008, 44: W09434

68 Yustres A, Asensio L, Alonso J, et al. A review of Markov Chain Monte Carlo and information theory tools for inverse problems in subsurface flow. Computat Geosci, 2012, 16: 1-20

69 Foglia L, Mehl SW, Hill MC, et al. Evaluating model structure adequacy: The case of the maggia valley groundwater system, southern switzerland. Water Resour Res, 2013, 49: 260-282

70 Ye M. MMA: A computer code for multimodel analysis. Ground Water, 2010, 48: 9-12

71 Rojas R, Feyen L, Batelaan O, et al. On the value of conditioning data to reduce conceptual model uncertainty in groundwater modeling. Water Resour Res, 2010, 46: W08520

72 Rojas R, Feyen L, Dassargues A. Sensitivity analysis of prior model probabilities and the value of prior knowledge in the assessment of conceptual model uncertainty in groundwater modeling. Hydrol Process, 2009, 23: 1131-1146

73 Troldborg M, Nowak W, Tuxen N, et al. Uncertainty evaluation of mass discharge estimates from a contaminated site using a fully Bayesian framework. Water Resour Res, 2010, 46: W12552

74 Raftery A E, Gneiting T, Balabdaoui F, et al. Using Bayesian model averaging to calibrate forecast ensembles. Mon Weather Rev, 2005, 133: $1155-1174$

75 Harp D R, Vesselinov V V. Analysis of hydrogeological structure uncertainty by estimation of hydrogeological acceptance probability of geostatistical models. Adv Water Resour, 2012, 36: 64-74

76 Post J, Hattermann F F, Krysanova V, et al. Parameter and input data uncertainty estimation for the assessment of long-term soil organic carbon dynamics. Environ Modell Softw, 2008, 23: 125-138

77 Renard B, Kavetski D, Kuczera G. Comment on "An integrated hydrologic Bayesian multimodel combination framework: Confronting input, parameter, and model structural uncertainty in hydrologic prediction" by Newsha K. Ajami et al. Water Resour Res, 2009, 45: W03603

78 Krzysztofowicz R. Bayesian theory of probabilistic forecasting via deterministic hydrologic model. Water Resour Res, 1999, 35: 27392750

79 Kavetski D, Kuczera G, Franks S W. Bayesian analysis of input uncertainty in hydrological modeling: 2. Application. Water Resour Res, 2006, 42: W03408

80 Kavetski D, G Kuczera, SW Franks. Bayesian analysis of input uncertainty in hydrological modeling: 1 . Theory. Water Resour Res, 2006, 42: W03407

81 Ebrahimi N, Soofi E S, Soyer R. Information measures in perspective. Int Stat Rev, 2010, 78: 383-412

Open Access This article is distributed under the terms of the Creative Commons Attribution License which permits any use, distribution, and reproduction in any medium, provided the original author(s) and source are credited. 